\title{
Plädoyer für eine unabhängige und grundrechtsgerechte Evaluation der EU-Richtlinie zur Vorratsdatenspeicherung
}

Christina Schlepper und Matthias Leese

\section{Der rechtliche Hintergrund}

Mit dem Gesetz zur Neuregelung der Telekommunikationsüberwachung und anderer verdeckter Ermittlungsmaßnahmen sowie zur Umsetzung der Richtlinie 2006/24/EG vom 21.12.2007 wurde die Vorratsdatenspeicherung in Deutschland implementiert. Darin wurde festgelegt, dass Telekommunikationsunternehmen Telefon- und Internetverbindungsdaten für sechs Monate speichern müssen. Aus diesen Daten geht hervor, wer mit wem wann und wie lange telefoniert hat und wer mit wem wann in E-mail- oder SMS-Kontakt stand. Bei der Handynutzung wird auch der Standort festgehalten. Diese Regelung resultiert aus der nationalstaatlichen Umsetzung der EU-Richtlinie 2006/24/ EG (Data Retention Directive, DRD) vom 15. März 2006 zur Speicherung von Daten, „die bei der Bereitstellung öffentlich zugänglicher elektronischer Kommunikationsdienste oder öffentlicher Kommunikationsnetze erzeugt oder verarbeitet werden “ (Amtsblatt der Europäischen Union 2006: L 105/54). Die Verkehrs- und Standortdaten müssen für einen Zeitraum von mindestens sechs Monaten und höchstens zwei Jahren gespeichert werden. Diese Speicherungsfrist gilt jedoch nicht für zu Ermittlungszwecken abgerufene Daten und kann von den Mitgliedsstaaten unter Angabe besonderer Umstände für einen begrenzten Zeitraum verlängert werden (ebd.: L 105/59). Die Befristung der Speicherung ist in diesem Sinne also als flexibel zu bezeichnen, was einer ernsthaften Begrenzung zuwiderläuft.

Die Verabschiedung der EU-Richtlinie zur Vorratsdatenspeicherung versteht sich als Reaktion auf die Anschläge auf das World Trade Center vom 11. September 2001 (Schlögel 2010: 168). Auch nach den Anschlägen von London betonte der Rat noch einmal, dass eine europäische Regelung der Vorratsdatenspeicherung notwendig sei (Amtsblatt der Europäischen Union 2006: L 105/55). Gestützt auf positive „wissenschaftliche Untersuchungen als auch praktische Erfahrungen in mehreren Mitgliedsstaaten“ (ebd.) mit Regelungen zur Speicherung von Telekommunikationsdaten, die zu einer erhöhten Ermittlung, Feststellung und Verfolgung von Straftaten geführt hätten, sahen das Europäische Parlament und der Rat die Notwendigkeit, die teilweise stark variierenden nationalstaatlichen Regelungen auf der supranationalen Ebene zu harmonisieren. Diese Harmonisierung wird allerdings nicht konsequent verfolgt und erstreckt sich allein auf die rechtlichen Aspekte; die technische Umsetzung wird auch weiterhin den Mitgliedsstaaten überlassen (ebd.: L 105/56).

Das Bundesverfassungsgericht (BVG) hat in seinem Urteil vom 2. März 2010 die deutsche Regelung zur Vorratsdatenspeicherung in ihrer konkreten Ausgestaltung für unzulässig erklärt. ${ }^{1}$ Der vom BVG vorgegebene Änderungsbedarf wurde von der Bundesregierung bisher nicht umgesetzt, weshalb seit dem Urteil in Deutschland keine Verkehrsdaten mehr gespeichert werden. Das Justizministerium unter der Leitung von Sabine Leutheusser-Schnarrenberger steht der Vorratsdatenspeicherung generell kritisch gegenüber (Schlögel 2010: 172) und hofft nach eigener Aussage auf eine Regelung der Leerstelle durch die supranationale Ebene.

Um eine Harmonisierung der unterschiedlichen nationalstaatlichen Umsetzungen zu erreichen, führt die Europäische Kommission zur Zeit eine Evaluation der Richtlinie 2006/24/EG durch. Das Instrument der Evaluation erfreut sich gerade bei umstrittenen Regelungen immer größerer Beliebtheit. ${ }^{2}$ In Deutschland ist die Evaluationsforschung Ende der sechziger Jahre aufgekommen, und die Ziele lagen zunächst in der Erhöhung der Rationalität politischer Entscheidungen, der Etablierung einer Rückmeldeschleife, dem Abbau von Überregulierung und Bürokratisierung sowie einer Stärkung der Kontrollfunktion des Parlaments (Lange 1999: 907). Grundsätzlich erscheint die Evaluation in der Gesetzgebungspraxis als sehr sinnvolle Maßnahme für den Gesetzgeber, um zu überprüfen, ob sich die getroffenen Regelungen als geeignet erwiesen haben.

Die Bewertung der Richtlinie zur Vorratsdatenspeicherung bezieht sich zum einen darauf, inwieweit durch die Richtlinie erreicht wurde, dass die Telekommunikationsdaten zum Zweck der Strafverfolgung zur Ver- fügung stehen. Zum anderen geht es in der Bewertung darum festzustellen, inwieweit sich die Vorratsdatenspeicherung auf nationaler Ebene als wirksam zur Bekämpfung von Kriminalität erwiesen hat (vgl. Bundesregierung 2009: 2). Das Room-Document der EU-Kommission (2010), welches als Entwurf des Evaluationsberichts ${ }^{3}$ angesehen werden kann, gibt Auskunft über dessen Ziele. Es diene dazu sicherzustellen, dass die Vorratsdatenspeicherung zur Kriminalitätsbekämpfung beitrüge und rechtmäßig sei. Das Evaluationsverfahren weist jedoch teilweise gravierende Mängel auf, und es kommen Zweifel an der Unabhängigkeit und Ergebnisoffenheit der Evaluation sowie der Bewertung der Angemessenheit der Richtlinie auf.

Wir zeigen in diesem Beitrag, dass das Evaluationsverfahren der Europäischen Kommission zur Richtlinie 2006/24/EG einer kritischen Überprüfung unter wissenschaftlichen Gesichtspunkten nicht standhalten kann. Um die Notwendigkeit einer adäquaten Durchführung der Evaluation zu unterstreichen, werden wir abschließend auf einige Ergebnisse aus dem DFG-Projekt „Der ,überwachte’ Bürger zwischen Apathie und Protest - zur Genese neuer staatlicher Kontrolltechnologien und ihren Effekten auf Einstellungen und Verhalten der Bevölkerung “ eingehen.

\section{Das Evaluationsverfahren der EU- Kommission}

Artikel 14 der Richtlinie („Bewertung“) verpflichtet die Kommission, dem Europäischen Parlament und dem Rat bis zum 15. September 2010 eine „Bewertung der Anwendung dieser Richtlinie sowie ihrer Auswirkungen auf die Wirtschaftsbeteiligten und die Verbraucher vor[zulegen], um festzustellen, ob die Bestimmungen dieser Richtlinie [...] gegebenenfalls geändert werden müssen “ (Amtsblatt der Europäischen Union 2006: L 105/60). Die Ergebnisse dieser Evaluation werden der Öffentlichkeit zugänglich gemacht. Das Evaluationsverfahren der Europäischen Kommission startete am 14. Mai 2009 mit einer Konferenz, an der 140 Vertreter von Interessengruppen aus den Straf- 
verfolgungs- und Datenschutzbehörden, der Wirtschaft, der Wissenschaft und der Zivilgesellschaft teilnahmen. Im Anschluss wurde der Entwurf eines Fragenkatalogs von Vertretern der Mitgliedstaaten und der Staaten des europäischen Wirtschaftsraums (EEA) diskutiert und an die beteiligten Akteure (Strafverfolgungsbehörden und Telekommunikationsbehörden der Mitgliedstaaten, Europäisches Parlament und zivilgesellschaftliche Akteure, Datenschutzbehörden und privater Sektor) übersandt. Bemerkenswerterweise fanden Vertreter aus der Wissenschaft nun keine Berücksichtigung mehr. Bis Januar 2010 konnte (nur) eine Rücklaufquote von 70 ausgefüllten Fragebögen verzeichnet werden, auf deren Grundlage der Entwurf des Evaluierungsberichts der EU-Kommission - das sog. ,Room Document' (EU-Kommission 2010) - entstand. Einige ausgefüllte Antwortfragebögen sowie das Room Document sind auf der Internetseite des AK Vorratsdatenspeicherung verfügbar. ${ }^{4}$

Der Fragenkatalog der EU-Kommission gliedert sich in vier Kapitel. Der erste Abschnitt, der sich an die Mitgliedstaaten richtet, befasst sich mit dem Umfang und den Modalitäten der Anwendung, den wirtschaftlichen Auswirkungen sowie der Wirksamkeit und Effizienz der Vorratsdatenspeicherung. Das zweite Kapitel des Fragenkatalogs beschäftigt sich mit den Auswirkungen der Vorratsdatenspeicherung auf Bürgerrechte und Maßnahmen zur Begrenzung der nachteiligen Auswirkungen. Fragen diesbezüglich wurden an das Europäische Parlament und Bürgerrechtsorganisationen gerichtet. Im dritten Abschnitt wird die Sichtweise nationaler Datenschutzbehörden sowie des Europäischen Datenschutzbeauftragten erhoben. Das letzte Kapitel des Fragenkatalogs ist an Kommunikationsdiensteanbieter des privaten Sektors adressiert. Für unseren Zusammenhang sind die ersten beiden Kapitel des Fragenkatalogs interessant.

\section{Kritik an der Evaluation durch die EU-Kommission}

Unsere Kritik an der Evaluation der Richtlinie zur Vorratsdatenspeicherung entzündet sich im Wesentlichen an vier Punkten: (1) an der fehlenden Unabhängigkeit und Ergebnisoffenheit der Evaluation sowie der unzureichenden Überprüfung der (2) Wirksamkeit, (3) Erforderlichkeit und (4) Verhältnismäßigkeit der Vorratsdatenspeicherung.

\section{(1) feblende Unabhängigkeit und Ergebnisoffenheit:}

Wissenschaftliche Mitglieder bzw. wissenschaftliche Expertise sucht man in der Expertengruppe (bestehend aus Vertretern der nationalstaatlichen Strafverfolgungsbehörden, Telekommunikationsverbänden, Datenschutzbehörden, dem Datenschutzbeauftragten der EU und Mitgliedern des Europäischen Parlaments) vergeblich. Vor dem Hintergrund des Spannungsverhältnisses zwischen sozialem Wandel und einer vergleichsweise statischen Gesetzgebung, die nicht (mehr) in der Lage ist, die Dynamiken der Moderne ausreichend flexibel, reaktionsfähig und differenziert zu verarbeiten (Schulze-Fielitz 1994: 140), ist gerade im Bereich der elektronischen Kommunikation eine unabhängige Bewertung von gesetzgeberischen Interventionen notwendig. Schnelle technische Entwicklungen, wie sie im Bereich der Speichermöglichkeiten von Kommunikation im Rahmen der Vorratsdatenspeicherung möglich geworden sind (und sich beständig weiter entwickeln werden), verschärfen den skizzierten Konflikt zusätzlich, da die technischen Regelungen von Interventionen schon innerhalb kürzester Zeit überholt sein können.

Aus methodischer Sicht ist die Abhängigkeit des Evaluators vom Auftraggeber der Evaluation als höchst problematisch zu betrachten. Der soziale und politische Kontext von Evaluationen sorgt häufig dafür, dass positive Bewertungen der Intervention erwartet werden, um damit die Zielpopulation, Kritiker, die Opposition oder auch die Öffentlichkeit davon zu überzeugen, dass die Maßnahmen angemessen und effektiv seien. Studien mit negativen Ergebnissen werden hingegen häufig nicht publiziert, da sie der gewünschten Legitimation der Intervention zuwiderlaufen (Lange 1999: 913). SchulzeFielitz (1994: 165) weist zusätzlich darauf hin, dass „die Ministerialbürokratie eher in ihrer eigenen Wirkungsoptik befangen sein und solche Wirkungen verfolgen [dürfte], die sie durch die gesetzliche Regelung intendieren wollte; ein sektoral spezialisiertes Fachreferat wird Nebenwirkungen schon außerhalb seines Zuständigkeitsbereichs eher vernachlässigen [...]. “

Es ist folglich generell kritisch zu hinterfragen, inwiefern Evaluationen ergebnisoffene Prozesse darstellen. Neben einer strukturellen Eingebundenheit kann gerade auch finanzielle Abhängigkeit vom Auftraggeber der Studie zu einer fraglichen Neutralität des Evaluators führen. Aus diesem Grund ist es entscheidend, dass eine Evaluation von unabhängiger Seite durchgeführt wird. Im Optimalfall sollte Evaluationsforschung also von externen Stellen durchgeführt werden; interne Evaluationen sind als unzureichend abzulehnen (Lange 1999: 914).

Aus dieser Konstellation ergeben sich auch Probleme, was die Akzeptanz und Umsetzung von Evaluationsergebnissen anbelangen. Gerade Ergebnisse, die finanziellen Handlungsbedarf implizieren und somit „unbequem“ für den Auftraggeber der Evaluation werden, laufen Gefahr, ebenso unter den Tisch zu fallen, da „mit Lern- und Veränderungseffekten im öffentlichen Bereich erfahrungsgemäß nur selten zu rechnen ist“ (ebd.). Aus diesem Grund sollte sowohl über die Evaluationskriterien als auch über die Umsetzung der Erkenntnisse bereits im Vorfeld ein Konsens bestehen. Auch hier weist die Evaluation der Richtlinie zur Vorratsdatenspeicherung einen gravierenden Mangel auf. Sie verpflichtet lediglich zur Durchführung der Bewertung, wie diese konkret auszugestalten ist, wurde im Vorfeld jedoch nicht festgelegt.

\section{(2) unzureichende Überprüfung der Wirksamkeit der Vorratsdatenspeicherung:}

Politische Maßnahmen sind Interventionen, die darauf angelegt sind, bestimmte (soziale) Problemfelder gezielt in eine gewünschte Richtung zu beeinflussen. Beispiele hierfür sind etwa die Erhöhung des Anteils weiblicher Beschäftigter im öffentlichen Sektor, die Reduktion von Umweltbelastungen oder, wie in unserem Zusammenhang, die Senkung von Straftaten bzw. die Erhöhung der Aufklärungsquote. Die Intervention überführt im besten Fall den Ist-Zustand in einen Soll-Zustand. Allgemein gesprochen, lässt sich als Untersuchungsgegenstand von Evaluationen die Diskrepanz zwischen intendierten Wirkungsprozessen und den faktisch feststellbaren, empirischen Auswirkungen von Interventionen bezeichnen.

$\mathrm{Zu}$ diesem Zweck identifizieren Rossi, Lipsey und Freeman (2004: 18) fünf zeitlich abgestufte Dimensionen, auf denen die Evaluation einer Intervention stattfinden kann: (1) die Notwendigkeit, (2) die konkrete Ausgestaltung, (3) die Implementation, (4) die Auswirkungen und (5) die Effizienz der Intervention. Die ersten beiden dieser Dimensionen weisen einen prospektiven Ansatz auf, der neben der Feststellung eines Regelungsbedarfs unter Berücksichtigung von alternativen Lösungsansätzen auch Kri- 
terien wie Betroffenenfreundlichkeit, Vollzugstauglichkeit oder das Kosten-NutzenVerhältnis berücksichtigen sollte. Auf diese Weise soll eine optimierte Ausgestaltung der Intervention erreicht werden (Bussmann 2006: 43).

Die drei weiteren Dimensionen der Evaluation besitzen einen retrospektiven Charakter. Neben der Bewertung der konkreten Umsetzung von abstrakten Interventionsrichtlinien stehen dabei vor allem die Auswirkungen der Intervention im Fokus. Effizienz lässt sich durch einen Abgleich von formulierten Zielen und messbaren Umsetzungen dieser Ziele feststellen. Probleme ergeben sich in der Praxis der Evaluationsforschung jedoch aus der Operationalisierung und Messung der in der Intervention benannten Ziele. Idealerweise wird eine entsprechende Konzeption deshalb schon bei der Planung der Intervention berücksichtigt (Lange 1999: 914). Dies ist wie bereits erwähnt bei der Richtlinie zur Vorratsdatenspeicherung nicht erfolgt.

Zur Erhebung der Wirksamkeit der Vorratsdatenspeicherung finden sich zwei Fragen im Fragenkatalog der Kommission. Zum einen wird die Frage gestellt, wie die Erfolgsquote der Verwendung von Vorratsdaten sei. Zum anderen wird gefragt, ob die Verwendung von Verkehrsdaten bei der gerichtlichen Feststellung und/oder Verfolgung von Straftaten hilfreich war, die sonst gescheitert wäre. Hierzu sollten Beispiele angeführt werden.

Zuerst einmal ist $\mathrm{zu}$ vermerken, dass in der Antwort der Bundesregierung (2009) quantitative Angaben zur Erfolgsquote der Verwendung von Vorratsdaten durchweg fehlen. Es heißt nur: „Statistiken über Erfolgsquoten werden nicht geführt" (ebd.: 15). Konkret zu beziffern ist lediglich im Umkehrschluss, dass für den Zeitraum vom 1.5.2008 bis 31.8.2009 ${ }^{5}$ in 63 Prozent der betroffenen Verfahren ein erfolgloses Auskunftsersuchen dazu führte, dass die Straftat nicht aufgeklärt werden konnte (vgl. ebd.: 16). Darüber hinaus werden zwei Fallbeispiele angeführt, in denen der Zugriff auf Vorratsdaten zum Ermittlungserfolg führte. Es erscheint jedoch höchst unzulässig, auf der Basis von zwei Einzelfällen auf eine generelle Wirksamkeit der Vorratsdatenspeicherung zu schließen und kann aus wissenschaftlicher Sicht nicht als Beleg der Effizienz gelten.
(3) feblende Überprüfung

der Erforderlichkeit der

Vorratsdatenspeicherung:

Eine Frage danach, ob auch mildere Mittel, d.h. weniger grundrechtsinvasive Maßnahmen zur Verbrechensaufklärung hätten führen können, ist im Fragenkatalog überhaupt nicht enthalten. Eine Überprüfung der Erforderlichkeit, wie sie Albers (2005: 31f.) als Kriterium einer grundrechtsgerechten Evaluation fordert, findet somit nicht statt.

Gerade angesichts der Eingriffsschwere, welche die Speicherung von Telekommunikationsdaten bedeutet, ist die Erhebungsweise der Wirksamkeit, aber auch der von der Bundesregierung erbrachte Nachweis der Notwendigkeit der Vorratsdatenspeicherung als äußerst dürftig zu betrachten. Dem steht auch der Befund gegenüber, dass sich die Aufklärungsquote für Straftaten insgesamt in den Jahren 2008 und 2009, als Verkehrsdaten gespeichert wurden, im Vergleich zu den Vorjahren 2005 bis 2007 ohne Vorratsdatenspeicherung kaum verändert hat. Im Bereich der Internet-Straftaten ist die Aufklärungsquote sogar gesunken (vgl. Arbeitskreis Vorratsdatenspeicherung 2010).

\section{(4) unzureichende Überprüfung der Verhältnismäßigkeit der Vorrats- datenspeicherung:}

Ein weiterer wichtiger Bestandteil für eine umfassende Evaluation ist die Untersuchung, wie sich die Intervention auf die Zielpopulation auswirkt. Bei dieser auch als impact evaluation bezeichneten Vorgehensweise sind nicht nur intendierte, sondern vor allem auch unintendierte Effekte zu berücksichtigen (Lange 1999: 909; Bussmann 2006: 43). In Bezug auf die Vorratsdatenspeicherung, die durch die Richtlinie 2006/24/EG eine Zielpopulation von über 500 Millionen Bürgern der Europäischen Union betrifft, ist die Analyse von unintendierten Wirkungsprozessen besonders wichtig. Auch Weinzierl (2006: 98) weist darauf hin, dass eine Evaluation sich nicht nur auf „eine Überprüfung der Effizienz im Sinne der Relation von Input und Output [...] [beschränken darf], sondern dass durch die Evaluation die Eingriffsintensität für Betroffene in ein Verhältnis zum Grad der Erreichung eines zuvor konkret genannten Gesetzesziels gesetzt [...] [werden muss].“ Die Überprüfung der Verhältnismäßigkeit eines sicherheitsbehördlichen Instruments ist somit als weiteres Kriterium zu fordern, das eine grundrechtsgerechte Evaluation zu erfüllen hat (vgl. Albers 2005: 32).

Der Fragenkatalog der EU-Kommission (2010) enthält eine Frage zu den Auswirkungen der Vorratsdatenspeicherung auf bürgerliche Freiheiten und verlangt nach Beispielen. Obwohl alleine die Antwort des AK Vorratsdatenspeicherung (2009) 35 Seiten umfasst und sehr differenziert zahlreiche empirische Belege dafür bringt, dass die Vorratsdatenspeicherung zu deutlichen Verhaltensänderungen in der Bevölkerung führt, wird im Entwurf des Evaluierungsberichtes (vgl. EU-Kommission 2010) nur in einem sehr kurzen Abschnitt darauf eingegangen, dass European Digital Rights und der AK Vorratsdatenspeicherung auf katastrophale Folgen für die Bürger hinweisen, die z.B. in steigenden Preisen, Wettbewerbs- und Freiheitsbeschränkungen sowie mangelndem Systemvertrauen bestünden. Nicht nur finden die negativen Auswirkungen auf die Bürger in der Zielsetzung der Bewertung keinen Platz, sondern auch ihre äußerst knappe Abhandlung im Entwurf des Evaluierungsberichtes erweckt den Eindruck, dass sie keine tatsächlich relevante Größe darstellen. Die Frage nach der Verhältnismäßigkeit, also ob der Nutzen der Vorratsdatenspeicherung im Verhältnis zu den Beeinträchtigungen steht, die durch die Maßnahme auf Seiten der Bürger unweigerlich entstehen, scheint in der Evaluation der EU-Kommission keine Rolle zu spielen. Dieses Bild verfestigt sich, wenn man betrachtet, wie die Alternativvorschläge der Bürgerrechtsorganisationen zur Begrenzung der nachteiligen Auswirkungen der Vorratsdatenspeicherung im Room Document berücksichtigt werden. Diese werden äußerst knapp und rein deskriptiv aufgelistet; eine darüber hinausgehende Auseinandersetzung findet nicht statt.

Um die Relevanz und Tragweite der Beeinträchtigungen, die die Vorratsdatenspeicherung für die Bürger bedeuten, zu untermauern, werden wir im folgenden Abschnitt auf einige Ergebnisse aus dem DFG-Projekt „Der ,überwachte' Bürger zwischen Apathie und Protest - zur Genese neuer staatlicher Kontrolltechnologien und ihren Effekten auf Einstellungen und Verhalten der Bevölkerung “ eingehen, das seit April 2009 am Institut für Sicherheits- und Präventionsforschung (ISIP) in Hamburg durchgeführt wird. 
Abbildung 1: Argumente der Oppositionsparteien gegen die Vorratsdatenspeicherung

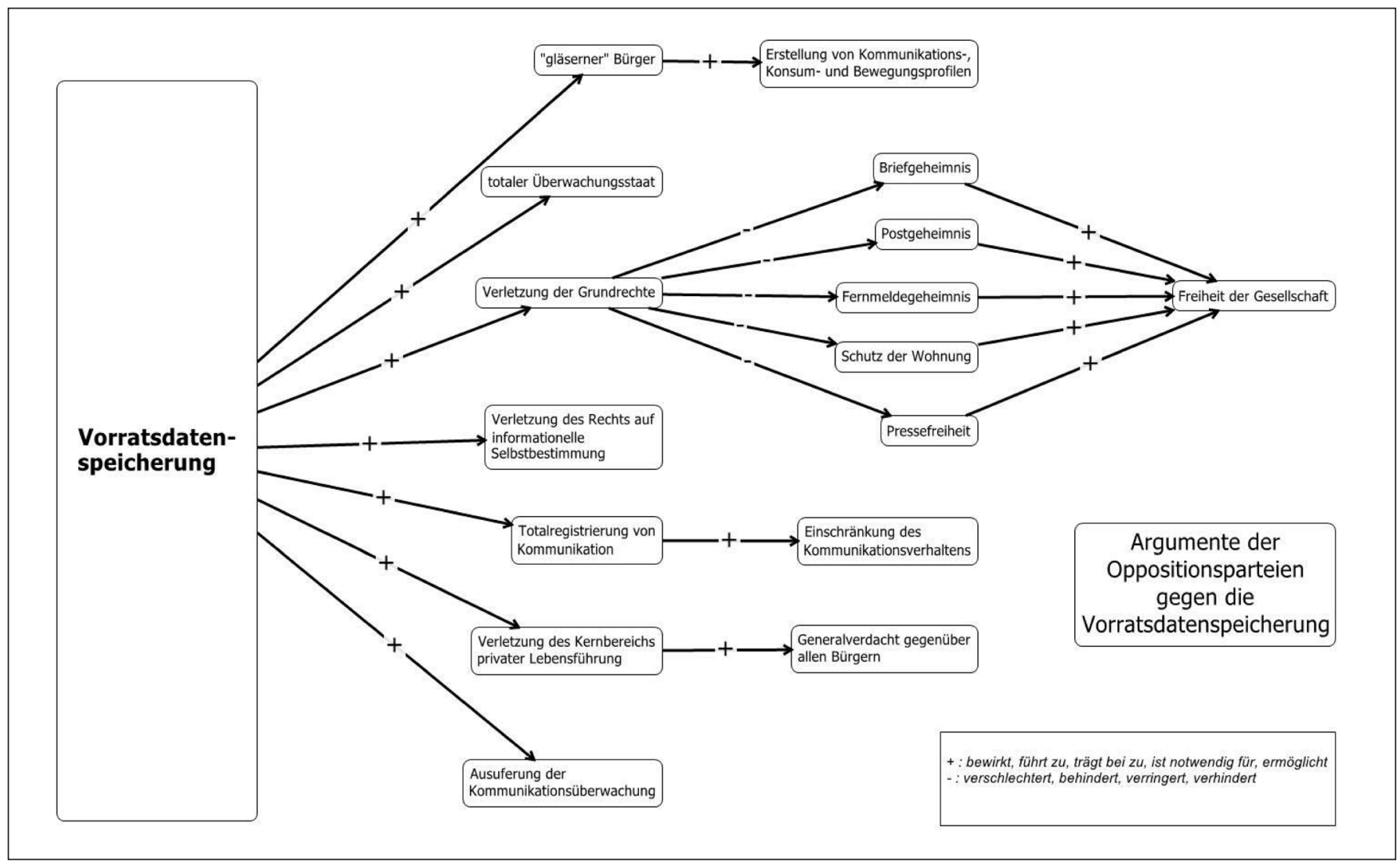

\section{Empirische Ergebnisse}

Abbildung 1 zeigt die Argumentationsketten, die Abgeordnete der Oppositionsparteien während des Gesetzgebungsverfahrens des Gesetzes zur Neuregelung der Telekommunikationsüberwachung und anderer verdeckter Ermittlungsmaßnahmen sowie zur Umsetzung der Richtlinie 2006/24/EG vom 21.12.2007 gegen die Einführung der Vorratsdatenspeicherung vorgebracht haben. $\mathrm{Zu}$ diesem Zweck haben wir alle Bundestags- und Bundesratsdrucksachen sowie die Plenarprotokolle analysiert, die den Gang der Gesetzgebung dokumentieren, und die Argumentationsketten in Form von Kausalpfeilen abgebildet. Die Kritik beschränkt sich nicht nur auf die rechtliche Ebene, indem auf die Verletzung von Grund- und Bürgerrechten hingewiesen wird. Es wird auch die Gefahr benannt, dass durch die Vorratsdatenspeicherung die Möglichkeit entsteht, dass Daten zur Erstellung von Kommunikations-, Konsum- und Bewegungsprofilen missbraucht werden können, möglich sind zudem Sozialprofile. Darüber hinaus wird sogar explizit auf die Verhaltensebene der Bevölkerung eingegangen und thematisiert, dass die Totalregistrierung der Kommunikation zur Einschränkung des Kommunikationsverhaltens der Bürger führen wür- de. Somit scheint zumindest in Teilen der politischen Reihen kein Zweifel daran zu bestehen, dass die Vorratsdatenspeicherung $\mathrm{zu}$ unerwünschten Verhaltensänderungen der Bürger führt. Ein eindrucksvolles Beispiel hierfür, das aus einer repräsentativen Forsa-Umfrage ( $\mathrm{N}=1.002$ ) aus dem Mai 2008 resultiert, führt der AK Vorratsdatenspeicherung (2009: 3) in seiner Antwort auf den Fragebogen der Europäischen Kommission an: „Die Mehrheit der Befragten würde wegen der Vorratsdatenspeicherung davon absehen, per Telefon, E-Mail oder Handy Kontakt zu einer Eheberatungsstelle, einem Psychotherapeuten oder einer Drogenberatungsstelle aufzunehmen, wenn sie deren Rat benötigten (517 der Befragten). Hochgerechnet entspricht dies über 43 Mio. Deutschen.“

Dass die Vorratsdatenspeicherung zu erheblichen Verhaltensänderungen der Bürger führt, hat sich auch in unserer standardisierten Telefonbefragung (CATI) ergeben. Diese wurde an einer repräsentativen Stichprobe $(\mathrm{N}=2.176)$ aus dem gesamten Bundesgebiet im Oktober 2009 durchgeführt. ${ }^{6}$ Die Erhebung fand also ebenfalls zu einem Zeitpunkt statt, an dem in Deutschland auf Vorrat gespeichert wurde. Im Mittelpunkt der Befragung standen neben der Vorratsdatenspei- cherung noch folgende weitere staatliche Kontrolltechnologien: biometrische Ausweisdokumente, der Online-Zugriff von Polizei- und Bußgeldbehörden auf digitalisierte Passbilder, das Kontenabrufverfahren, die Antiterrordatei sowie die Passagierdatenspeicherung. Verhaltensänderungen wurden zwar für alle diese Maßnahmen zusammengefasst erhoben, allerdings beinhalten die Antwortvorgaben eine Reihe von Items, die sich auf das Kommunikationsverhalten der Befragten beziehen und damit klar im Kontext der Vorratsdatenspeicherung stehen. Hierzu haben wir gefragt, welche der folgenden Handlungen vom Befragten schon einmal praktiziert wurden $(\mathrm{Ja}=1$; Nein $=$ $0)$ :

(1) zu einer Telefongesellschaft gewechselt, die Telefon- bzw. Internetverbindungsdaten nicht auf Vorrat speichert; (2) ein abhörsicheres Telefon bzw. Handy benutzt; (3) ein Programm oder einen Anonymisierungsdienst zum anonymen Surfen im Internet genutzt; (4) ein Programm zur Verschlüsselung von E-Mails oder Dateien eingesetzt; (5) ein Programm zur Erzeugung von Passwörtern verwendet; (6) eine E-Mail-Adresse mit falschem Namen angelegt; (7) um abhörsicher zu telefonieren, Internet-Telefonie, z.B. 
Tabelle 1: Häufigkeit der Items zum Kommunikationsverhalten

\begin{tabular}{|l|c|c|c|c|}
\hline \multirow{2}{*}{ Aktivität } & \multicolumn{2}{|c|}{ praktiziertes Verhalten } & \multicolumn{2}{c|}{ intendiertes Verhalten } \\
\cline { 2 - 5 } & $\mathrm{N}$ & $\%$ & $\mathrm{~N}$ & 888 \\
\hline Kryptographie nutzen & 461 & 27,5 & 63,1 \\
\hline Programm für Erzeugung von Passwörtern nutzen & 316 & 18,8 & 629 & 37,8 \\
\hline Email mit falschem Namen schreiben & 269 & 15,9 & 384 & 22,8 \\
\hline Internet-Telefonie (z.B. Skype) nutzen & 253 & 15 & 631 & 37,7 \\
\hline Anonymisierungsprogramm nutzen & 216 & 12,8 & 559 & 33,6 \\
\hline zu Provider ohne Vorratsdatenspeicherung wechseln & 125 & 6,4 & 1016 & 53,4 \\
\hline Internet-Café besuchen & 86 & 5,1 & 331 & 19,5 \\
\hline abhörsicheres Handy oder Telefon nutzen & 105 & 5 & 578 & 27,7 \\
\hline
\end{tabular}

Die Zahlen belegen, dass die Auswirkungen der Vorratsdatenspeicherung auf das Kommunikationsverhalten der Bürger keineswegs zu vernachlässigen sind, sondern dass ein nicht unerheblicher Anteil von Personen bereits auf andere Kommunikationsformen ausweicht oder Vorkehrungen trifft, um die Registrierung zu vermeiden. Gerade wenn man die rechte Spalte von Tabelle 1 betrachtet, wird deutlich, dass die Tendenz mutmaßlich steigt, alternative Kommunikationswege zu nutzen, um anonym kommunizieren zu können. Teilweise sogar über die Hälfte der Befragten hatten zum Zeitpunkt der Befragung die Intention, zu einem Provider zu wechseln, der Telefon- und Internetverbindungsdaten nicht auf Vorrat speichert oder ein Programm zur Verschlüsselung von E-Mails oder Dateien einzusetzen. Immerhin über ein Drittel der Befragten würde ein Programm für die Erzeugung von Passwörtern, Internet-Telefonie (z.B. Skype) oder ein Anonymisierungsprogramm nutzen. Eine derartige Veränderung des Kommunikationsverhaltens der Bürger kann auch aus Sicht des (europäischen) Gesetzgebers nicht wünschenswert sein und würde die Vorratsdatenspeicherung letztlich ad absurdum führen, weil sie ihren eigentlichen Zweck nicht mehr erreichen könnte, wenn eine solch extensive Nutzung alternativer Kommunikationswege stattfände.

Tabelle 2: Bewertung staatlicher Kontrolltechnologien

\begin{tabular}{|c|c|c|c|c|c|}
\hline \multirow{2}{*}{\multicolumn{2}{|c|}{ Staatliche Kontrolltechnologien }} & \multicolumn{4}{|c|}{ Antwortkategorien } \\
\hline & & sehr schlecht & eher schlecht & eher gut & sehr gut \\
\hline \multirow{2}{*}{ Vorratsdatenspeicherung } & $\mathrm{N}$ & 719 & 776 & 475 & 188 \\
\hline & $\%$ & $33,3 \%$ & $36,0 \%$ & $22,0 \%$ & $8,7 \%$ \\
\hline \multirow{2}{*}{ Online-Durchsuchung } & $\mathrm{N}$ & 535 & 632 & 655 & 330 \\
\hline & $\%$ & $24,9 \%$ & $29,4 \%$ & $30,4 \%$ & $15,3 \%$ \\
\hline \multirow{2}{*}{ Zugriff auf Bankdaten } & $\mathrm{N}$ & 265 & 703 & 808 & 378 \\
\hline & $\%$ & $12,3 \%$ & $32,6 \%$ & $37,5 \%$ & $17,5 \%$ \\
\hline \multirow{2}{*}{ Erfassung von Passagierdaten } & $\mathrm{N}$ & 192 & 527 & 971 & 462 \\
\hline & $\%$ & $8,9 \%$ & $24,5 \%$ & $45,1 \%$ & $21,5 \%$ \\
\hline \multirow{2}{*}{ Zugriff auf Passbilder } & $\mathrm{N}$ & 160 & 345 & 1044 & 598 \\
\hline & $\%$ & $7,5 \%$ & $16,1 \%$ & $48,6 \%$ & $27,9 \%$ \\
\hline \multirow{2}{*}{ biometrische Ausweisdokumente } & $\mathrm{N}$ & 155 & 323 & 979 & 691 \\
\hline & $\%$ & $7,2 \%$ & $15,0 \%$ & $45,6 \%$ & $32,2 \%$ \\
\hline \multirow{2}{*}{ Antiterrordatei } & $\mathrm{N}$ & 139 & 364 & 1037 & 604 \\
\hline & $\%$ & $6,5 \%$ & $17,0 \%$ & $48,4 \%$ & $28,2 \%$ \\
\hline
\end{tabular}

Dass die Vorratsdatenspeicherung die staatliche Kontrolltechnologie ist, die von den Befragten am negativsten gesehen wird und die Befragten keineswegs allen staatlichen Kontrolltechnologien so kritisch gegenüberstehen wie der Vorratsdatenspeicherung, ergibt sich aus Tabelle 2. Biometrische Ausweisdokumente etwa werden vom Großteil der Befragten positiv beurteilt. Auf die Frage wie gut bzw. wie schlecht sie die einzelnen staatlichen Kontrolltechnologien finden, antwortete ein Drittel, dass sie die Vorratsdatenspeicherung sehr schlecht finden. 36 Prozent bewerten sie mit eher schlecht. Somit wird die Vorratsdatenspeicherung (sogar mit einigem Abstand vor der Online-Durchsuchung) am stärksten und mehrheitlich von den Befragten abgelehnt.

Skype, genutzt; (8) um anonym zu surfen, ein Internet-Cafe besucht.

Zusätzlich wurde nach der Intention gefragt, diese Handlungen (auch) in Zukunft zu praktizieren (, Ja, das würde ich unter Umständen [wieder] tun “ = 1; „Nein, das würde ich unter keinen Umständen [wieder] tun “ $=0$ ).
Tabelle 1 gibt die absoluten $(\mathrm{N})$ und relativen $(\%)$ Häufigkeiten für die einzelnen Verhaltensänderungen wieder. Die Prozentangaben zu folgenden Aktivitäten beziehen sich nur auf Internetnutzer $(\mathrm{N}=1.699)$ : „Kryptographie nutzen“, „Programm für die Erzeugung von Passwörtern nutzen“, „E-Mail mit falschem Namen schreiben“,
„Internet-Telefonie (z.B. Skype) nutzen“, „Anonymisierungsprogramm nutzen“, „Internet-Café besuchen“. Alle übrigen Prozentzahlen beziehen sich auf die gesamte Stichprobe $(\mathrm{N}=2.176)$. 


\section{Fazit}

Es ist zwar generell als positiv zu bewerten, dass die Verankerung von Evaluierungspflichten im Zusammenhang mit sicherheitspolitischen Instrumenten in verstärktem Maße Einzug in die Gesetzgebungspraxis findet. Damit sich daraus für den Gesetzgeber aber tatsächlich eine kritische Überprüfung einer gesetzgeberischen Maßnahme ergibt, aus der sich Handlungsempfehlungen ableiten lassen, ist die Beachtung wissenschaftlicher Kriterien bei der Konzeption und Durchführung der Evaluation unbedingt erforderlich. Die Evaluation der Richtlinie 2006/24/EG durch die EU-Kommission ist aus mehreren Gründen als unzureichend abzulehnen, die wir abschließend noch einmal zusammenfassen möchten:

(1) Bei der Verabschiedung der Richtlinie wurde zwar die Durchführung einer Evaluation vorgesehen, allerdings fehlen im Vorfeld festgelegte Bestimmungen, nach welchen Kriterien die Evaluation durchzuführen ist. Durch diese fehlende Regelung existieren weder eine plausible Operationalisierung der Kriterien, unter denen der „Erfolg“ der Richtlinie gemessen werden sollte, noch wie mit den Ergebnissen der Evaluation umzugehen ist. Um aussagekräftige Ergebnisse zu erzielen, ist eine präzise Regelung der Konzeption einer Evaluation schon im Gesetzeslaut unumgänglich.

Augenfällig wird dieser Mangel, wenn etwa die deutsche Bundesregierung keine Zahlen zu den Erfolgsquoten der Vorratsdatenspeicherung bei der Aufklärung von Straftaten mit vorlegen kann. Stattdessen werden zwei Einzelfallbeispiele aufgeführt, in denen Vorratsdaten zur Aufklärung beigetragen hätten (vgl. Bundesregierung 2009: 16). Eine Verallgemeinerung auf der Basis von zwei Einzelfällen ist aus wissenschaftlicher Sicht als unzulässig zurückzuweisen.

(2) Die EU-Kommission hat zwar den Versuch unternommen, alle betroffenen Interessengruppen in den Feedback-Prozess einzubeziehen, eine wissenschaftliche Begleitung des Verfahrens fehlt allerdings. Da keine externen und unabhängigen Wissenschaftler hinzugezogen wurden, handelt es sich um eine interne Evaluation. Interne Evaluationen sind jedoch aus den skizzierten Gründen abzulehnen: sie tendieren dazu, fachbereichsspezifisch die Wirksamkeit von Interventionen zu bestätigen, ohne Nebeneffekte auf andere Bereiche adäquat zu berücksichtigen - zumal in diesem Fall die supranationale Ebene die Effizienz einer von ihr selbst vorgegebenen Richtlinie untersucht und kaum eingestehen wird, dass die Vorgaben für die nationalstaatliche Umsetzung nicht angemessen sind.

(3) Vertreter der Zivilgesellschaft (Bürgerrechtsorganisationen) wurden zwar befragt. Die umfangreichen Expertisen, die einen erheblichen negativen Einfluss der Vorratsdatenspeicherung auf Rechte und Verhalten der EU-Bürger attestieren, wurden jedoch in 14 Zeilen abgehandelt (vgl. EU-Kommission 2010: II. 1.), was nicht auf eine ernsthafte Berücksichtigung von unintendierten $\mathrm{Ne}$ beneffekten schließen lässt, deren Existenz aber zweifellos auch durch unsere empirischen Ergebnisse belegt wird.

(4) Dies ist umso erstaunlicher, da zumindest in der deutschen Politik die Auswirkungen der Vorratsdatenspeicherung auf bürgerliche Freiheitsrechte, das generelle Vertrauen in den Staat und das Kommunikationsverhalten der Bürger offensichtlich bekannt sind (vgl. Abb. 1). Warum diese Erkenntnisse, die die Frage nach der Verhältnismäßigkeit und Erforderlichkeit der Vorratsdatenspeicherung unweigerlich aufwerfen, nicht stärker in den Evaluationsprozess auf supranationaler Ebene eingegangen sind, ist nicht nachvollziehbar.

Insgesamt weist das Evaluationsverfahren der EU-Kommission daher schwerwiegende Defizite auf. Es scheint, dass die Bemühungen der supranationalen Exekutive eher darauf ausgelegt sind, für eine höchst umstrittene Sicherheitsmaßnahme im Nachhinein Akzeptanz und Legitimation zu schaffen. Von einer unabhängigen und grundrechtsgerechten Evaluation, die zu tatsächlich belastbaren Ergebnissen führt und einer kritischen Überprüfung standhalten kann, ist das Evaluationsverfahren der EUKommission jedoch weit entfernt.

Dipl. Soz. Dipl. Krim. Christina Schlepper, Institut für Sicherheits- und Präventionsforschung (ISIP), Bogenallee 11, 20144 Hamburg,E-mail: isip@uni-hamburg.de

Matthias Leese, B.A., Institut für Sicherheits- und Präventionsforschung (ISIP), Bogenallee 11, 20144 Hamburg, E-mail: isip@ uni-hamburg.de

\section{Literaturverzeichnis}

Albers, Marion (2006): Die verfassungsrechtliche Bedeutung der Evaluierung neuer Gesetze zum Schutz der Inneren Sicherheit. In: Albers, Marion (Hg.): Menschenrechte - Innere Sicherheit - Rechtsstaat. Konferenz des Deutschen Instituts für Menschenrechte ; Berlin, 27. Juni 2005. Berlin: Dt. Inst. für Menschenrechte (Dokumentation / Deutsches Institut für Menschenrechte).

Arbeitskris Vorratsdatenspeicherung (2009): Antworten auf den Fragebogen der Europäischen Kommission vom 30.09.2009 zur Vorrats- datenspeicherung. Online verfügbar unter http://www.vorratsdatenspeicherung.de/ images/antworten_kommission_vds_200911-13.pdf, zuletzt geprüft am 19.10.2010.

Bundesregierung (2009): Fragenkatalog zur Bestandsaufnahme hinsichtlich der Wirkungsweise der Richtlinie über die Vorratsspeicherung von Daten, die bei der Bereitstellung öffentlich zugänglicher elektronischer Kommunikationsdienste oder öffentlich zugänglicher elektronischer Kommunikationsnetze erzeugt oder verarbeitet werden, und zur Änderung der Richtlinie 2002/58/EG. 30.9.2009. Online verfügbar unter http://www.vorratsdatenspeicherung.de/images/DR-consult/de ms_de.pdf, zuletzt geprüft am 19.10.2010.

Bussmann, Werner (2006): Gesetzesevaluation und experimentelle Gesetzgebung, in: ius.full $1 / 06$, S. $42-52$.

EU-Kommission (2010): Room Document. Evaluation of Directive 2006/24/EC and of $\mathrm{Na}$ tional Measures to Combat Criminal Misuse and anonymous Use of Electronic Communications. Online verfügbar unter: http://www. vorratsdatenspeicherung.de/images/RoomDocumentEvaluationDirective200624EC. pdf, zuletzt geprüft am 19.10.2010.

Lange, Elmar (1999): Evaluationsforschung. In: Albrecht, Günter/Groenemeyer, Axel/Stallberg, Friedrich W. (Hg.): Handbuch Soziale Probleme, Opladen.

Rossi, Peter H./Lipsey, Mark W./Freeman, Howard E. (2004): Evaluation: A Systematic Approach. Thousand Oaks u.a.

Schlögel, Martina (2010): Die Karlsruher Entscheidung zur Vorratsdatenspeicherung: Lässt das Bundesverfassungsgericht die Bürger im Regen stehen? In: Gesellschaft, Wirtschaft, Politik, H. 2/2010, S. 167-174.

Schulze-Fielitz, Helmuth (1994): Zeitoffene Gesetzgebung. In: Hoffmann-Riem, Wolfgang (Hg.): Innovation und Flexibilität des Verwaltungshandelns. 1. Aufl. Baden-Baden: Nomos-Verl.-Ges. (Schriften zur Reform des Verwaltungsrechts, 2).

Weinzierl, Ruth (2005): Die Befristung und Evaluierung von Sicherheitsgesetzen: ein wirksames Instrument des Menschenrechtsschutzes und der Wahrung rechtsstaatlicher Prinzipien. In: Deile, Volkmar; Hutter, Franz-Josef; Kurtenbach, Sabine, et al. (Hg.) (2005): Freiheit in Gefahr - Strategien für die Menschenrechte. 1. Aufl., Orig.-Ausg. Frankfurt am Main: Suhrkamp. S. 93-99

\section{Fußnoten:}

1 Zur Urteilsbegründung: http://www.bundesverfassungsgericht.de/pressemitteilungen/bvg10011 (Stand: 20.10.2010).

2 Auch andere Sicherheitsgesetze wie z.B. das Terrorismusbekämpfungsgesetz vom 9.1.2002, das Gemeinsame-Dateien-Gesetz vom 22.12.2006 und das Gesetz zur Abwehr von Gefahren des internationalen Terrorismus durch das Bundeskriminalamt (BKA-Gesetz) vom 25.12.2008 wurden mit einer Evaluierungsklausel versehen.

3 Eigentlich hätte der fertige Evaluationsbericht dem Europäischen Parlament spätestens am 15.9.2010 vorgelegt werden müssen. Die Frist wurde nun mit dem Verweis auf fehlende Daten aus den Mitgliedstaaten auf Dezember 2010 verschoben. Daher kann hier nur auf das Room Document Bezug genommen werden.

4 http://www.vorratsdatenspeicherung.de/

5 In diesem Zeitraum fand eine Sondererhebung statt, die durch einstweilige Anordnungen des Bundesverfassungsgerichts veranlasst wurde.

6 Grundgesamtheit: alle Personen über 18 Jahre in deutschen Privathaushalten mit Festnetzanschluss. Screening: Geburtstagsmethode. 